ISSN: $2344-8636$

2 (2): $97-104$

\title{
Transformando la Práctica del Cuidado desde el Propio Conocimiento de la Disciplina de Enfermería
}

\section{Transforming the Nursing Practice from the Proper Knowledge of the Discipline of Nursing \\ Recibido: 14 Mayo 2014/Enviado para modificación: 2 de Julio 2014/Aceptado: 17 de Julio 2014}

\section{Cenith Cabarcas Rodríguez ${ }^{1}$}

Universidad Simón Bolívar

\section{RESUMEN}

La enfermería ha evolucionado a partir de los acontecimientos y las corrientes de pensamiento que han surgido a lo largo de la historia. Para conocer esta evolución, es necesario abordar los diferentes paradigmas y concepciones que han ido configurando su desarrollo en conjunto con otras ciencias. En consecuencia, es importante infundir en el estudiante la comprensión de los cambios que se están produciendo desde el propio conocimiento de la disciplina, lo cual le permitirá adaptarse y prepararse para enfrentar los nuevos retos y desafíos a partir de la interacción con los seres humanos, así como ser partícipe activo en la transformación de la práctica.

Palabras Clave: Práctica, cuidado, conocimiento, disciplina, enfermería (Fuente: DeCS).

\section{ABSTRACT}

Different paradigms and conceptions based on thoughts and events that have arisen throughout history have taken part in the evolution of nursing along with other sciences. Consequently, it is important to make students aware of the changes that are taking place based on the available nursing know-how and knowledge in order to adapt and prepare themselves to face new challenges as they interact with human beings, as well as become active participants in the nursing evolution.

Keywords: Patient care, knowledge, discipline, nursing (Source: MeSH, NLM).

Para citar este artículo: Cabarcas C. Transformando la Práctica del Cuidado desde el Propio Conocimiento de la Disciplina de Enfermería. Cienc. innov. salud. 2014; 2 (2):97 - 104

\footnotetext{
${ }^{1}$ Enfermera. Magister en Enfermería. Universidad Simón Bolívar. Correo electrónico: ccabarcas8@ unisimonbolivar.edu.co
} 


\section{Introducción}

En las últimas décadas, las enfermeras y enfermeros han contribuido al desarrollo de la disciplina con el aporte de nuevos modelos y teorías de gran utilidad en la práctica clínica, lo que enriquece a la vez el quehacer y el lenguaje enfermero. La utilidad de un modelo conceptual depende del valor que se le da y la forma en que se le emplea. Además, esa misma utilidad permite recuperar una identidad profesional que ha sido cuestionada y actúa como un importante factor para determinar los procesos e indicadores de la práctica (1).

Por otra parte, para el desarrollo de cualquier campo del conocimiento, es fundamental comprender su evolución: la forma cómo se acumula y cómo se acepta el mismo, pues, tal comprensión contribuye a la definición de metas a seguir, ya sea individual o colectivamente, para la disciplina como un todo (2).

Cabe resaltar que los modelos y teorías de enfermería se aplican para facilitar su desarrollo en la investigación, educación, administración y práctica clínica (3). De igual forma, las teorías en enfermería se han convertido en un tema importante durante los últimos cincuenta años, estimulando el crecimiento y la expansión de la educación y la literatura que da soporte a la disciplina. En este sentido, en la evolución de la enfermería, juega un papel clave la bibliografía resultante de la investigación de enfermeros y enfermeras académicas y clínicas con el ánimo de mejorar la práctica del cuidado.

La búsqueda del conocimiento sustantivo ha llevado a los estudiosos de la disciplina a identificar teorías que guíen la práctica y la investigación. Asimismo, la disciplina ha seguido una trayectoria que la ha llevado a conceptos y marcos conceptuales, a modelos y teorías y, últimamente, a la teoría de rango medio (4).

Precisamente, aun cuando se conoce que las teorías creadas entre los años sesenta y los ochenta contribuyeron al fortalecimiento de los currículos de enfermería, hoy por hoy se desconoce casi en su totalidad los aportes que ha hecho la teoría de rango medio, la cual es muy aplicable a la práctica, y ayuda desde la academia a la comprensión de los fenómenos del cuidado. En concordancia con lo anterior y de acuerdo con la revisión de literatura sobre este último tipo de teoría de rango medio, se sabe que los constructos inspirados en ella se encuentran entre los menos abstractos y, al mismo tiempo, son los más prácticos, dado que especifican características de situaciones de enfermería (4).

El origen de las teorías de mediano alcance se atribuye al sociólogo Norton, en cuya disciplina surgieron y luego fueron adoptadas por la enfermería en el año 1974. En este campo se evidenció su pertinencia para guiar la investigación empírica (5).

Como ya hemos expresado, durante los últimos cincuenta años, las teorías en enfermería se han convertido en una tarea importante. A pesar de ello, aún existe poco conocimiento por parte de los profesionales en enfermería en el desarrollo y la aplicación de estas teorías. De hecho, en la actualidad, desde la práctica clínica, se puede identificar el fenómeno de la no utilización y aplicación de la teoría en enfermería. En esta disciplina, se lleva una práctica muy empírica, en que se puede identificar muy poco el propio conocimiento de la enfermería.

En todo caso, las teorías de rango medio proporcionan un abordaje prometedor para fortalecer teoría, investigación y práctica, pero exigen hacer mayor énfasis en su desarrollo y utilización para sostener la investigación y la práctica de la enfermería (6).

Entre las teorías de rango medio que han sido desarrolladas en la pasada década, se encuentran: la teoría de la incertidumbre en la enfermedad de Merle Mishel, el modelo de interacción de Cox, la teoría de los síntomas desagradables de Lenz, el modelo de constelación de la enfermedad de Morse y Johnson, y el modelo de Pender. Este último ha tenido un gran auge en el programa de enfermería de la Universidad Simón Bolívar de Barranquilla, debido a su impacto en el cuidado de las personas desde la promoción de la salud, que apuntan a los objetivos del milenio en materia de salud pública.

El objetivo de este artículo de reflexión se enfoca, entonces, en destacar la importancia de las teorías de enfermería y el acervo de conocimiento disciplinar que guía el quehacer enfermero, el enlace que este debe tener entre la práctica y la investigación. Esto en razón de que, desde la academia, resulta necesario esclarecer los conceptos, así como su utilidad y aplicación. 


\section{Desarrollo}

La enfermería se fundamenta en los resultados de investigación y en el conocimiento de la práctica diaria, todo ello sustentado en la filosofía e historia de la disciplina (4).

Si bien es cierto, la investigación en enfermería ha seguido un curso irregular y de altibajos, urge la necesidad de hacer investigación con el fin de dar respuestas y plantear la solución de problemas persistentes. Esto último en el entendimiento de que la meta final de esta profesión consiste en mejorar la práctica de sus miembros, de modo que los cuidados brindados a sus pacientes tengan la mayor eficacia (7).

En efecto, si la práctica de enfermería ha de ser más que un acto basado en rituales y mitos, si ha de ser una práctica profesional, entonces la investigación debe ser su cometido principal, pues solo esta puede generar, refinar y aumentar el conocimiento científico que se requiere para llevar a esta disciplina hacia un verdadero estatus profesional (8).

Por consiguiente, la enfermería debe estudiar los fenómenos relevantes que pueden ser observados en individuos, grupos, situaciones y eventos, valorando la realidad y, de esta manera, hacer aportes que impacten de manera positiva en la calidad del cuidado. Lo anterior no debe estar suelto de la teoría, pues, desde la academia, cada vez urge la necesidad de estimular al estudiante a la utilización de la teoría en la investigación y en la práctica.

Ahora bien, desde hace varios años y a partir de las ciencias sociales, como la antropología, la sociología y la psicología, la investigación cualitativa se ha convertido en una vía de conocimiento de creciente relevancia en las investigaciones relacionadas con la salud (9). Esta metodología constituye una salida en pro del desarrollo de la disciplina y de la construcción de la enfermería como profesión social, tomando en cuenta que el cuidado del ser humano, en sus dimensiones individual y colectiva, es su objeto de estudio (10), pero se ha establecido de manera muy desigual (11).

Cabe resaltar que desde los inicios de la enfermería, se tuvo en cuenta la formación vocacional. La enfermería prestaba servicios, un cuidado excelente a sus pacientes, pero muchas de sus prácticas no fueron probadas ni se usaron con uniformidad, aunque parecían eficaces (9). El proceso de transformación de la práctica toma tiempo y compromiso, pues, entre otras cosas, dicha práctica se centra en una perspectiva normativa, que está condicionada por el diagnóstico médico y el tratamiento (12).

Y es claro que la enfermería es una disciplina práctica, pero, precisamente por ello, las ideas innovadoras deben surgir de la práctica aunque con una buena fundamentación teórica, la cual pueda fortalecer los conocimientos sobre el cuidado, en relación con las necesidades de la persona, familia o comunidad a la cual se ofrece. De esta manera, las teorías son verificadas y validadas a través de la investigación y proporcionan una orientación para esa investigación que, a su vez, le da un toque de innovación desde el cuidado.

La práctica de la enfermería ha sido siempre el objetivo principal propuesto por los líderes de la profesión de la enfermería, por tal razón se requiere una reflexión académica desde su propio conocimiento. Lo paradójico es que el conocimiento en enfermería ha generado elementos de suma importancia que han sido poco explorados, como es la situación de la narrativa del cuidado, pues, se ha establecido que todo conocimiento de la enfermería reside en la situación de enfermería, que, a su vez, es el depósito y el contexto del conocimiento de enfermería (13).

En concordancia con lo anterior, Bárbara Carper definió los patrones de conocimiento en la situación de enfermería, los cuales residen de manera implícita en la misma disciplina, perspectiva que aparece como un cambio filosófico emergente desde el enfoque epistemológico (8).

Con base en los patrones de Carper, Silva afirma que hay aspectos del conocimiento de enfermería inexplicables y desconocidos, que llevan a preguntarse acerca de cómo conocer lo desconocido y darle significado y cómo conocer lo inexplicable y hallarle sentido (14).

Lo anterior conlleva a plantear la necesidad de explorar un cambio emergente para la disciplina a través de la educación; y de esta manera, comprender desde la crítica la importancia de ese cambio emergente, dando a conocer a los estudiantes el arte y la ciencia del cuidado.

En este sentido, desde la propia experiencia docente, se ha aportado a la construcción de los microcurrículos 
del programa de enfermería, introduciendo patrones de conocimiento como son: el empírico, el estético, el ético y el personal, los cuales constituyen en sí el conocimiento propio de la disciplina. En todos ellos se visualiza al ser humano como un todo, según la perspectiva del metaparadigma de enfermería. Al respecto, Watson afirma que: "Los modelos y teorías de enfermería se fundamentan en una visión humanista del cuidado. El cuidado es para la práctica de enfermería su razón moral, no es un procedimiento o una acción, el cuidar es un proceso interconectado, intersubjetivo, de sensaciones compartidas entre la enfermera y el paciente" (1).

El cuidado humano debe basarse en la reciprocidad y debe ser único y auténtico. Los enfermeros son llamados para ayudar al paciente y aumentar su armonía entre la mente, el cuerpo y el alma, para generar procesos de conocimiento de sí mismo (15). Cuidar del otro, según Mayeroff, es ayudar a crecer a ese otro, ya se trate de una persona, una idea, un ideal, un trabajo de arte o una comunidad. Y ayudar a otras personas a crecer, también significa animarlas y asistirlas para que sean cuidadas por alguna cosa o por otra persona aparte de nosotros mismos (12).

El cuidado es una forma de expresión, de relación con el otro ser y con el mundo, es una forma de vivir plenamente. El cuidar implica comportamientos y acciones que envuelven conocimientos, valores, habilidades y actitudes emprendidas para favorecer las potencialidades de las personas, para mantener o mejorar la condición humana en el proceso de vivir y morir. Cuidar implica comprender y ser comprendido, buscando el crecimiento y desarrollo de la persona, del ser en el mundo. El cuidado es "como una forma ética y estética de vivir, que se inicia por el amor a la naturaleza y pasa por la apreciación de lo bello" (16).

Es decir, la enfermera y el enfermero no solo deben adquirir conocimiento científico prestado de las otras ciencias como anatomía, fisiología, psicología y administración. También deben adquirir un conocimiento propio de la disciplina de enfermería, que se deriva del día a día, en su práctica del cuidado con los demás. Así es como se pueden lograr transacciones de cuidado con los demás y ejercer realmente el arte de la enfermería.

De igual forma, la enfermería debe desarrollar una conceptualización propia, que le permita dar respuestas al cuidado para un verdadero cambio paradigmático. Con otras palabras, debe centrarse en el campo de la investigación, de la teoría y la práctica, de forma que se consigan verdaderos aportes al desarrollo de los currículos con sus respectivas conceptualizaciones.

Ahora bien, la dificultad que ha tenido la enfermería para afianzar los cambios curriculares de acuerdo con conceptualizaciones propias, quizás se deba a la estructura de los sistemas de prestación de servicios, que son una réplica del modelo médico, donde la mayoría de acciones de salud se enmarcan en un enfoque curativo, en programas de acciones verticales basados en un patología específica (17).

Al reflexionar sobre esto último, se evidencia la necesidad de que el estudiante y futuro profesional de enfermería se vea a sí mismo como una persona cuidadora, que logre comprender su propia esencia de ser humano y sea capaz de conocer y comprender al otro como cuidador. Una experiencia así lleva a expresar de manera auténtica el cuidado tanto a quien cuida como a quien es cuidado. De igual forma, se debe reconocer el acervo del propio conocimiento para la contribución a la calidad de vida de las personas y al mejoramiento de la salud.

Le enfermería entra así en el campo de la persona cuidada, con la intención de conocer al otro, de reconocerlo como persona, y, a través de esta interacción, permite que ese otro reconozca a quien lo cuida como persona.

Justamente, desde hace aproximadamente tres años, en el programa de enfermería de la Universidad Simón Bolívar (Barranquilla, Colombia), la situación de enfermería se ha convertido en uno de los elementos centrales del micro currículo para lograr la enseñanza y el reconocimiento del cuidado en nuestros estudiantes, generando así una presencia auténtica y una respuesta efectiva en el cuidado.

$\mathrm{Y}$, en este orden de ideas, un punto para destacar desde los procesos educativos es la interrelación entre teoría, práctica e investigación. Estas tres cuestiones, al integrarse, dan a la enfermería rumbos claros que se reflejan en la práctica. En otras palabras, la interrelación de estos tres elementos fortalece el conocimiento propio fundamentado en el cuidado, pero también fortalece el conocimiento enfermero, puesto que la enfermería es una disciplina y cuenta con un cúmulo de conocimientos que ha permitido su desarrollo. 
Por otra parte, a través de la investigación, se desarrolla y genera conocimiento para la enfermería, lo que, a su vez, fortalece la práctica del cuidado y de esta manera se valida la teoría enfermera. Esta triada se convierte en un elemento clave para el desarrollo de la profesión, ya que una disciplina es específica de una escuela y hace referencia a una rama de la educación, a un departamento de aprendizaje, a un dominio de conocimientos (18).

Morse (19) desarrolló un análisis comparativo de las teorías de cuidado, cuyo resultado fue la conceptualización desde los propios asertos desarrollados por las diferentes teorías. Fue así como aparecieron las categorías o perspectivas de cuidado, las cuales han permitido hoy por hoy una clasificación clara sobre la esencia de la enfermería. A través del ejercicio académico y desde la formación curricular del programa de Enfermería de la Universidad Simón Bolívar, esta esencia ha sido fundamental para la formación integral del estudiante desde el propio conocimiento y es la base para la conceptualización del cuidado, el cual incluye hoy perspectivas como: trato humano, imperativo moral, cuidado como afecto, cuidado como interacción interpersonal y cuidado como intervención terapéutica, la experiencia de la subjetividad y la respuesta física del paciente.

Por otra parte, se entiende como profesión a un campo especializado de la práctica, creado sobre la estructura teórica de una ciencia, así como sobre el conocimiento de esa disciplina y las habilidades que la acompañan. De modo que la estructura de la disciplina empieza a ajustarse cuando se reorganizan los conceptos de muchas teóricas en enfermería, puesto que sus concepciones encajan en un patrón más grande de la disciplina, cuyo resultado se resume en conceptos centrales que se describen a continuación (7):

Salud: El intento de la relación: Donde la salud es central para la disciplina de enfermería, el concepto de bienestar y de enfermedad o, como en nuestro medio se resume, el proceso salud-enfermedad.

Cuidado: La naturaleza de la relación: El cuidado es también uno de los términos centrales para la identidad de la profesión. En este sentido, Neuman argumentó que la enfermería es un facilitador de la salud y que el cuidado constituye una cualidad de la relación que potencia una relación trasformativa entre la enfermera y el paciente.
Conciencia: El concepto de conciencia se traduce en un concepto de expansión de la conciencia, que incluye una total información del campo (envolvimiento de la enfermera-paciente), es decir, el envolvimiento de un todo: sensaciones, emociones e intenciones.

Proceso mutuo: Se refiere a la manera en que la relación se desarrolla. El proceso mutual ocurre en el momento en que la enfermera logra estar muy presente con el individuo, la familia y la comunidad. En el proceso mutual, el individuo es invitado a participar través de las reflexiones y oportunidades para nueva conciencia, acciones, movimientos y transformaciones en su vida.

Patrón: La atención a los patrones es un aspecto central paras la práctica y la investigación en enfermería. El propósito de la atención a los patrones en enfermería involucra la explicación del conocimiento de la disciplina. Los patrones revelan el envolvimiento de la naturaleza en su totalidad. Newman se refiere al reconocimiento de patrones, los cuales se enfocan hacia el significado de la relación de eventos en la vida de las personas, cuyo proceso requiere de acciones potenciales.

Presencia: Se traduce en la resonancia de la relación. El concepto de presencia también puede establecerse como diálogo genuino, compromiso, apertura hacia el otro, atención y lo trascendental. Esto demuestra la autenticidad de la relación enfermera-paciente y la relación mutua y recíproca.

Por último, el Significado: Que juega un papel importante en la relación enfermera-paciente, pues, la presencia del cuidado y la resonancia de las enfermeras y enfermeros debe impactar en la vida de las personas que cuidan.

El significado se puede abordar desde diferentes áreas del saber: la filosofía, con enfoques metodológicos, la fenomenología, la hermenéutica, la lingüística, la pedagogía, la psicología social y la etnografía, entre otras. Estas diversas formas de concebir el conocimiento conllevan a distintos abordajes que dan soporte a la práctica (20).

La teoría guía los microcurrículos y da coherencia al lenguaje enfermero, al proponer herramientas a las académicas en enfermería y, por ende, a la práctica profesional para generar la habilidad y guiar la práctica como situación-producción. Por otra parte, 
guía el lenguaje común enfermero entre profesionales en enfermería, teniendo en cuenta sus concepciones meta- paradigmáticas (persona, salud, entorno $\mathrm{y}$ enfermería), que aparecieron en la década de los ochenta, un periodo importante en la teoría de enfermería elaborada por Jackeline Fawcett. Estas concepciones ofrecieron una perspectiva para la práctica, la administración, la educación, la investigación y el desarrollo posterior de las teorías en enfermería. Por lo tanto, el conocimiento de la persona, salud y el entorno en enfermería forman la base para el reconocimiento de la enfermería como disciplina y este conocimiento se les debe enseñar a aquellos que forman parte de la profesión.

De igual forma, en la profesión de enfermería, la teoría permite fomentar la autonomía, ayuda a dignificar al ser humano, tanto a quien cuida como a quien es cuidado, genera respuestas asertivas y efectivas en el cuidado del otro, permite desarrollar un cuerpo de conocimientos para guiar los diagnósticos y planes de enfermería, así como la relación del cuidado enfermero-paciente, $y$, finalmente, define alcances de la práctica, filosofía y misión de las organizaciones, generando así efectividad y una verdadera transformación en el cuidado (21).

Todo lo anterior constituye un pilar para ejercer una práctica idónea que permita cambios en los escenarios hospitalarios y el desarrollo moral de la persona, esto es: respeto y devoción, deber profesional, conocimiento, ciencia y tecnología, firmeza, valentía y voluntad de actuar frente a situaciones que se presenten en el día a día.

Es de suma importancia analizar dos aspectos en los currículos de enfermería; el concepto de cuidado como punto central y por otra parte, el proceso del ciclo vital humano. Un estudio sobre los avances en el cuidado de enfermería del grupo de cuidado de la Universidad Nacional de Colombia determinó que al interrelacionar estas dos variables, se propicia, en efecto, una reflexión sobre la importancia de la formación, y deja claro que esta debe centrarse en una creencia filosófica y en valores del cuidado. Asimismo, reclama la comunicación del grupo de enfermeras y enfermeros, particularmente de docentes (1).

Por tal razón, se necesita generar más cohesión entre la práctica y la academia. Articuladas estas con la investigación, se fortalece desde los convenios docencia-servicio una coherencia del lenguaje común enfermero que favorece la práctica y a las personas a quienes se les brinda cuidado.

Por otra parte, Pinto Afanador, en su texto "El cuidado como objeto de la práctica de enfermería", recalca la necesidad de dar un significado a la disciplina profesional de enfermería, que requiere en concreto generar conocimiento propio para resolver los problemas de la práctica y facilita la validación del conocimiento disciplinar. Esta misma autora plantea la urgencia de generar y validar teorías de tipo descriptivo y prescriptivo, la realización de investigaciones científicas y la cualificación de la práctica (21).

\section{Conclusiones}

En las academias de enfermería, se debe generar el interés en utilizar el conocimiento desde la formación en las aulas, lo cual debe ser trascendental para el estudiante. Solo de esta manera se lograrán avances en el desarrollo disciplinar.

El proceso de transformación de la práctica toma tiempo, pues esta aún tiene una perspectiva normativa direccionada por un diagnóstico médico. Para entender y comprender el cuidado, se debe vivir en él cuidado y crecer en él. El núcleo de la disciplina de enfermería, es decir, el cuidado, requiere el entendimiento de la relación persona a persona (13).

Por ello, la verdadera transformación de la práctica se logra desde la comprensión del cuidado como camino de crecimiento tanto para el que cuida como para quien es cuidado, es allí donde se visualiza una relación de mutualidad, el conocimiento y la comprensión. Además, esto genera el desarrollo del patrón personal del conocimiento en enfermería, ya que así el profesional logra comprender y actuar frente a la situación y a la persona que espera y solicita el cuidado.

Las enfermeras y enfermeros deben reflexionar más sobre el "conocer" que sobre el "hacer", aspecto este que ha prevalecido desde los comienzos de la enfermería profesional. Si una disciplina práctica refleja una práctica repetitiva, fundamentada en la rutina y en la tradición no se requiere de la teoría, pero si se desea una práctica innovadora basada en el conocimiento científico como debería ser toda práctica profesional, y con claro conocimiento por parte de las profesionales del significado de ella $y$ sus 
intervenciones, entonces la teoría sí que es necesaria (22).

De igual forma, las nuevas tendencias en la enfermería se deben comprender a partir de su propio contexto, puesto que el desconocimiento del mismo ha dificultado el desarrollo de esta ciencia y ha evitado la aplicación de la teoría en la práctica, lo cual es el eje central del avance de la disciplina (23).

En concordancia con todo lo anterior, es indiscutible la necesidad de comprender la utilidad de la teoría en enfermería. Los autores enfatizan en que para el futuro de la disciplina y de la ciencia de enfermería se debe: primero, comprender el verdadero papel que juega la teoría para el avance de una disciplina práctica; y segundo, manejar y utilizar la teoría de enfermería y otras teorías en su justo requerimiento (24).

\section{Referencias}

1. Sánchez B. Utilidad de los modelos conceptuales de enfermería en la práctica. En: El arte y la ciencia del cuidado. Grupo de cuidado Facultad de enfermería. Bogotá; 2010. Universidad Nacional de Colombia.

2. Saewyc E. Nursing theories of caring. A paradigm of adolescent nursing practice. $\mathrm{J}$ holist Nurs. 2013; 18(2): 114-128.

3. Cisneros F. Teorías y modelos en enfermería. Fundamentos de enfermería. Universidad del Cauca[Internet].2013 [citado 14 de mayo de 2013].Disponible en: http://biblioms.dyndns.org/Libros/Enfermeria/Teo riasYModelosDeEnfermeriaYSuAplicacion.pdf

4. Marrimer A, Alligoth M. Importancia de las teorías para la disciplina. En: Modelos y Teorías en enfermería. $7^{a}$ EDICIÒN. Elsevier. España. 2011.

5. Blegen MA, Tripp-Reimer T. Implications of nursing Taxonomies for Midle Rangue theory. ANS Adv Nurs Sci. 1997; 19(3): 37-49.

6. Lenz, E et al. Las teorías de rango medio. Adv. in Nursing Science.1995; 17(3): 1-13.

7. Boykin A, Shoenhofer SO, Jhon B. Living Caring practice. Transformative Power of the Theory of nursing as Caring. International Journal for Human Caring. 2005; 9 (3): 15-19.

8. Carper, B. De los patrones de conocimiento de Carper a las formas de ser: Un cambio filosófico y deontológico en enfermería. Adv. in Nursing Science.1995; 18 (1).1-3.

9. Boykin, A., Shoenhofer, SO. En: Enfermería como cuidado: Un modelo para transformar la práctica. Capitulo 3.ALN. NY, 1993. Traducido por: Sánchez, Beatriz. La situación de enfermería como punto central. Universidad Nacional de Colombia.

10. Watson, J. Nursing: The Philosophy and Science of Caring. Colorado: Colorado Associated University Press; 1985.

11. Morse JM et al. La investigación cualitativa: ¿realidad o fantasía? En: Asuntos críticos en investigación cualitativa. Colección Contus Universidad de Antioquia, Facultad de enfermería.2006.p. 241.

12. García M, Gomes B, Rojas A, Becerril L. Cuidado de la salud: Paradigma del personal de enfermeros en México. La reconstrucción del camino. Esc Anna Nery Rev de enferm AbrilJunio; 13 (2):287-96. Disponible en: http://www.scielo.br/pdf/ean/v13n2/v13n2a08.pdf

13. Fundamentos conceptuales de la profesión de enfermería. Manual educativo ANEC, folleto 5. Teoría de enfermería. Bogotá: 1990. p.196.

14. Gómez OJ, Daza LA, Zambrano VM. Percepción de cuidado en estudiantes: Caracterización e impacto para la formación y la visión del ejercicio profesional. Avenferm [Citado el 14 de agosto de 2014] ; 26 (1): 85-96.

15. Neuman B, Fawcett J. Neuman Sistems Model. 4 Edition. Upper Saddle River, Nj: Prentice Hall; 2002.

16. Garzón N. Ética en el cuidado de enfermería. En: Dimensiones del cuidado Grupo de cuidado. Bogotá. Unibiblos; 1988.

17. Duran M. Enfermería, Desarrollo teórico e investigativo. Universidad Nacional de Colombia. Capítulo 1. Santa fé de Bogotá, Unibiblos; 1998.

18. Polite D, Hungler B. Investigación científica en ciencias de la salud.Interamericana McGrawHill.Mexico; 1991. p. 563.

19. Morse JM, Bottorf J, Neander W, Solberg W. Comparative Analysis of Conceptualizations and theories of caring. Revista IMEGE. Journal Of nursing scholarship. 1991; 23(2): 1-3.

20. Parrado Y, Caro V. Significado, conocimiento para la práctica de enfermería. Avenferm. 2008 XXVI (2): 116-125. [citado el 22 de agosto de 2014]. 
21. Pinto N. El cuidado como objeto del conocimiento de enfermería. Avan enferm.2002 .V (1) 20 pp. 43-51. [Citado el 22 de agosto de 2014]

22. Meleis, AI. Theoretical Nursing: Development and Progress. $5^{\circ}$ edition. Philadelphia: WoltersKlumer/ Lippincott Williams \& Wilkins; 2012.

23. Duran M.La teoría, soporte de la ciencia y práctica de enfermería: Tendencias. Avenferm. 2012. XXX (1): 9-12. [Citado el 24 de marzo de 2014]. Disponible en: http://www.indexf.com/rae/301pdf/009012.pdf

24. Fundamentos conceptuales de la profesión de enfermería. Manual educativo ANEC, folleto 5. Teoría de enfermería. Bogotá: 1990. p.196.. 